\title{
Disponibilidad léxica en niños de 6 años. Alcance y proyección didáctica del corpus léxico infantil
}

Lexical availability of 6-year-old children. Scope and didactic projection of children's lexical corpus

\section{BEGOÑA GÓMEZ DEVÍS}

UNIVERSITAT DE VALÈNCIA

ORCID: http://orcid.org/0000-0001-8829-9833

RESUMEN: Nuestro propósito es presentar, en el marco de la Disponibilidad Léxica, los estudios dedicados a edades tempranas (niños de 6 años) revisando el alcance que este tipo de trabajos aporta al desarrollo de la programación y planificación de la enseñanza de lenguas. Para ello se ofrece una breve incursión en las bases de la disciplina, se comparan los resultados de índole cuantitativa obtenidos en España (Gran Canaria y Valencia) y Chile (Talca), y se aborda el análisis de las relaciones asociativas generadas en los inventarios de léxico disponible. Concluimos que los corpus de léxico disponible infantil proporcionan bases rigurosas para programar el léxico que deben aprender los escolares de los primeros cursos de Primaria tanto en aspectos de riqueza y precisión, así como en la configuración del lexicón mediante redes asociativas.

Palabras clave: disponibilidad léxica, léxico infantil, redes semánticas, corpus, enseñanza de lenguas

ABSTRACT: Our aim is to present -in the frame of Lexical Availability- the studies dedicated to early ages, 6-year-old children, examining the scope that this kind of works give to the development of programming and planning the teaching of languages. For this purpose, a short exploration on the bases of discipline is offered; quantitative results obtained in Spain (Gran Canaria and Valencia) and in Chile (Talca) are compared; and the associative relationships generated in the available lexical inventories are analysed. We conclude that the available lexical corpus of children provide accurate bases to programme the 
vocabulary that schoolchildren in the first years of Primary School must learn, both in terms of richness and accuracy as well as in terms of lexicon formation using associative networks.

Key words: lexical availability, children vocabulary, semantic networks, corpus, teaching of languages

\section{INTRODUCCIÓN}

Acometer el estudio del léxico disponible en edades tempranas supone un gran avance en el entramado de la disponibilidad léxica (DL) panhispánica. Como indican recientes investigaciones vinculadas a los niveles educativos básicos o primarios de ambos lados del Atlántico (Cepeda, Granada y Pomes, 2014; Gómez-Devís, 2019; Henríquez, Mahecha y Mateus, 2016; Hernández, 2014; Jiménez, 2015a, 2015b; Prado y Galloso, 2008; Samper, 2009; Samper, Hernández y Samper 2019, 2020) ello entraña complementar el conocimiento teórico sobre esta rama de la léxico-estadística afianzando los corpus de léxico disponible de la comunidad o variedad lingüística investigada y, de manera específica, considerar aspectos relevantes como la edad o el sexo, entre otros, para explicar posibles cambios en el caudal léxico, así como aportar nuevos materiales para la enseñanza de la lengua española en los diferentes niveles educativos planificación y selección-.

Si bien esta parcela indagatoria cuenta con escasa bibliografía, especialmente si la comparamos con los trabajos dedicados a otras etapas educativas -bachillerato o secundaria-, contar con investigaciones que comparten pautas metodológicas en el estudio del léxico disponible infantil permitirá abordar en un futuro próximo la progresión -riqueza y precisión- del vocabulario de los estudiantes de distintos niveles educativos.

En este sentido, no cabe duda del alcance que para la programación escolar presenta el conocimiento del vocabulario que los niños traen consigo, qué palabras han incorporado a su competencia léxica. Así lo señalaba López Morales al afirmar que la escuela no tiene por qué enseñar vocabulario ya aprendido y que los primeros materiales para la enseñanza de la lectura y la escritura deberían contemplarlo.

Sabemos que entre la edad de año y medio y los siete años, el vocabulario del niño aumenta rápidamente hasta llegar a las 2000 o 2500 unidades. Este es el promedio de adquisición léxica que tiene un niño normal al ingresar a la escuela. Pero ¿cuáles son esas palabras? [...] La forma de conseguir esa información es mediante la preparación de listas de frecuencia y a través de investigaciones de disponibilidad léxica en la población infantil.

(López Morales, 1986: 78)

Por otro lado, todas las escuelas lingüísticas están de acuerdo en que el léxico se organiza mentalmente de alguna manera, descartando categóricamente que se trate de una mera acumulación de palabras a la manera de los diccionarios. También se acepta que las representaciones del lenguaje comprenden la información léxica, basada en la forma componentes fonológico, morfológico y ortográfico-, y la información semántica-rasgos de la definición semántica de la palabra con los asociados más comunes- (Hunt y Ellis, 2004). Dicho esto, resulta palmario comprender que ello implica, especialmente en el proceso de enseñanza-aprendizaje, la identificación de categorías de percepción del 
discurso y de secuencias de vocablos concretos con las respectivas probabilidades de aparición en el discurso (Ellis, 2011: 48).

Así pues, este trabajo aborda la construcción de un corpus léxico infantil generado desde la DL - escolares de 6 años- indagando su alcance y proyección en la didáctica de la lengua. Nuestro principal propósito es facilitar una información básica, aunque reducida, de los aspectos teóricos y metodológicos que sustentan este tipo de investigaciones, así como el análisis contrastivo de los resultados obtenidos en tres estudios recientes (Cepeda, Granada y Pomes, 2014; Gómez-Devís, 2019; Samper, Hernández y Samper, 2019, 2020). En otras palabras, se pretende contribuir al desarrollo de la competencia léxica en edades tempranas ajustando la práctica didáctica no solo desde una óptica cuantitativa, sino también con marcado carácter cognitivo. Para ello se intentará dar respuesta a cuestiones como ¿Cuál es el promedio de respuestas por informante? ¿Cómo se relacionan las unidades en los repertorios de léxico disponible infantil? ¿En qué categorías o subcategorías se integran?

En definitiva, el interés por descubrir cómo se organiza el lexicón de los escolares en distintas etapas de su vida académica es el primer paso para lograr una práctica didáctica ajustada, de calidad, y orientada a la construcción de redes. Lograr este reto exige establecer las dimensiones del vocabulario disponible en diferentes cursos o etapas del proceso de enseñanza-aprendizaje. Dichos datos favorecerán la asunción de criterios objetivos para la selección y gradación del léxico en los diferentes niveles curriculares.

\section{BASES DE LA INVESTIGACIÓN EN DISPONIBILIDAD LÉXICA}

La DL nació el siglo pasado vinculada a la enseñanza de lenguas segundas. A mediados del siglo XX, con el propósito de favorecer la adquisición del francés en las colonias, se vio la necesidad de fijar el léxico elemental (Gougenheim, Michéa, Rivenc y Sauvageot, 1956) y poco más tarde el léxico fundamental ${ }^{1}$ (Gougenheim, Michéa, Rivenc y Sauvageot, 1964). Desde aquellas investigaciones primigenias hasta las actuales los cambios y reajustes metodológicos han sido numerosos y de gran calado, no obstante, todavía se persigue el objetivo de catalogar y clasificar el léxico indisolublemente ligado a temas o situaciones específicas - thématique ${ }^{2}$-; esto es, aquellas palabras, principalmente sustantivos, que son consideradas tópicos del tema en cuestión o pertinentes y que sin duda son necesarias en el intercambio comunicativo de un contexto concreto.

Aunque los inicios de esta modalidad indagatoria se dan en Francia y Canadá, es en el ámbito de la lengua española donde se conforma una vasta literatura y numerosos equipos de investigación, de ambos lados del Atlántico, la abordan con Humberto López Morales (1973) como máximo exponente. A partir de los años 90 se constata una excelente trayectoria gracias a los estudios publicados en distintos países de habla hispana que permiten el análisis y contraste del léxico disponible desde una perspectiva sociolingüística (sexo, lugar de residencia, nivel sociocultural, tipo de centro educativo, etc.) o cognitiva (L1, L2, LE), dando paso a la confección del diccionario panhispánico de léxico disponible. Asimismo, en esta última década se ha ampliado su marco de estudio

\footnotetext{
${ }^{1} \mathrm{Si}$ bien los primeros estudios toman como único criterio la frecuencia al considerar que las palabras de uso más frecuente son las más importantes para su enseñanza, poco tiempo después el análisis de dichos recuentos muestra la necesidad de incorporar el léxico de carácter potencial, aquel que solo aparece cuando la situación comunicativa lo precisa. En otras palabras, la suma de ambos (léxico frecuente y potencial) constituye el léxico fundamental de una lengua.

2 Es ese tipo de vocabulario que, a diferencia del frecuente o atemático -presente en cualquier acto comunicativo-, aparece solo cuando el tema o la situación comunicativa lo requieren.
} 
a otras lenguas peninsulares como el gallego y el catalán (Gómez-Devís y Llopis, 2016; Llopis y Gómez-Devís, 2010; López y Álvarez de la Granja, 2014; Serrano, 2014, 2020).

En cuanto a las cuestiones de índole metodológica, y dado que existen suficientes publicaciones $^{3}$ que consideran con gran detalle el conjunto de exigencias y parámetros asumidos por el magno proyecto panhispánico, nos limitaremos a dar una síntesis. En los estudios iniciales la recogida de datos consistía en solicitar a escolares que anotaran 20 palabras relacionadas con un centro de interés concreto (Gougenheim, Rivenc, Michéa y Sauvageot, 1964). Poco después, Dimitrijević (1969) propuso reajustar el sistema y recoger todas las palabras que viniesen a la mente de los informantes en un tiempo limitado. En el marco panhispánico actual el informante tan solo dispone de dos minutos para confeccionar una lista particular de vocablos asociados a un centro de interés -área nocional-. La encuesta de DL recoge, en primer lugar, los datos sociológicos del informante $\mathrm{y}$, a continuación, la información léxica relacionada con cada uno de los centros de interés observados ${ }^{4}$. Posteriormente, las soluciones aportadas por todos los participantes en las respectivas áreas temáticas son evaluadas mediante una fórmula estadística que calcula el índice de disponibilidad de cada unidad léxica en función de la frecuencia y posición en los listados producidos por los informantes, así como en relación con los datos sociológicos observados (López Chávez y Strassburguer, 1991).

Por otro lado, el hecho de contar con más de 45 equipos de investigación internacionales que comparten objetivos y método posibilita la reunión y el contraste de resultados entre numerosos países y zonas geográficas. En este sentido, son habituales las aportaciones con datos esencialmente cuantitativos y destacado carácter sociolingüístico: índice de disponibilidad (ID), promedio de unidades léxicas diferentes por centro de interés, total de palabras actualizadas en cada área nocional, índice de cohesión, promedio de respuestas por informante, incidencia y estratificación de las variables sociológicas en los repertorios de léxico disponible -sexo, tipo de centro educativo, lugar de residencia, nivel sociocultural, etc.-.

Otro aspecto intrínseco al ámbito de la DL es prestar atención a la vertiente cognitiva de los centros de interés, a la capacidad asociativa de las palabras. De acuerdo con Hernández y Tomé (2017: 106-108), la realización de la encuesta, también llamada test de disponibilidad, es una actividad de producción lingüística a partir de un estímulo artificial propiciado por un proceso mental ${ }^{5}$. Primero se identifica el estímulo, después, se activan varias representaciones mentales que propagan la activación de sus correspondientes nodos léxicos de las lenguas que conoce el hablante, se elige el nodo con el nivel de activación más alto $\mathrm{y}$, finalmente, se produce la palabra. Tras su producción, dicha palabra no se desactiva, sino que determina la selección de la palabra siguiente, con la que se relaciona de alguna manera. De hecho, Aichitson (1987: 72-85) ya señaló una arquitectura cerebral interactiva donde la jerarquización alcanza tanto a los

\footnotetext{
${ }^{3}$ Para mayor detalle se recomienda la consulta de Samper y Samper (2006), Paredes (2012) o Herranz (2019).

${ }^{4}$ Todos los trabajos que se incardinan en el Proyecto Panhispánico de Léxico Disponible deben atender los 16 estímulos o centros de interés tradicionales, aunque es posible ampliarlos según las particularidades de la investigación. Son los siguientes: Cuerpo humano, Ropa, La casa, Muebles de la casa, Alimentos y bebidas, Objetos colocados en la mesa, La cocina, La escuela, Iluminación, calefacción y ventilación, La ciudad, El campo, Medios de transporte, Trabajos del campo y del jardín, Animales, Juegos y distracciones, Profesiones y oficios.

5 Este proceso mental es fruto de tres procesadores: conceptualizador, formulador y articulador. El primero viene condicionado por la propia experiencia, el mundo exterior y la relación que tienen los sujetos con él. El segundo, etapa propiamente lingüística, aborda la transformación del mensaje preverbal al código lingüístico, se seleccionan las unidades que mejor se ajustan al contenido. Por último, el articulador, se ocupa de organizar los distintos motores que intervienen en la evocación.
} 
conceptos como al conjunto de asociaciones de tipo fonético, ortográfico, categorial, personal, etc. y comentaba, además, su carácter inestable, dado que los primeros pueden crear nuevas conexiones o modificar las existentes.

Dichas asociaciones o redes asociativas conforman un sistema fluido y dinámico, en constante modificación motivada por las nuevas informaciones que se procesan. La cantidad de asociaciones de cada palabra es variable: algunas asociaciones están fijadas por convenciones lingüísticas -campos semánticos, morfosemánticos, temáticos, léxicos, asociativos- $\mathrm{y}$ otras son personales.

Por último, confeccionar la lista de palabras - disponibles- en cada uno de los estímulos o centros de interés supone al hablante un esfuerzo y una actualización diferentes según la naturaleza del área temática en cuestión, las características lingüísticas y extralingüísticas de los informantes, y las estrategias utilizadas durante la realización del test de disponibilidad.

\subsection{EL LÉXICO DISPONIBLE DE LOS NIÑOS DE 6 AÑOS}

Tal como se ha apuntado al inicio, los estudios dedicados al léxico disponible en edades tempranas no son numerosos, pero se han jalonado a lo largo de más 30 años. Aunque es evidente que algunos de ellos presentan cierta pluralidad metodológica, especialmente los más antiguos, todos merecen su reconocimiento en la construcción del corpus de léxico disponible infantil. Entre las posibilidades que ofrecen dichos trabajos se subraya la relación directa entre la cantidad y calidad de palabras que un niño posee y los procesos asociados al conocimiento y comprensión del mundo que le rodea. Se señala, también, que sus resultados pueden ejercer como indicadores del desarrollo cognitivo dando cuenta de las posibilidades que los escolares encuestados tendrán para leer y escribir de manera efectiva, así como establecer la evolución o progreso del vocabulario según la edad de los informantes.

Es a partir de 1991, tras el desarrollo de la nueva fórmula léxico-estadística para calcular el índice de disponibilidad de López Chávez y Strasburguer, cuando su presencia se incrementa exponencialmente. Nos referimos, por ejemplo, a los estudios dedicados al contexto mexicano entre los que destacan López Chávez (1993) y Hernández Solís (2014); también los trabajos chilenos de Echeverría (1991) y Cepeda, Granada y Pomes (2014). Sin olvidar las aportaciones de Murillo $(1993,1994)$ y Sánchez y Murillo (1999, 2006) en Costa Rica o el estudio sobre los escolares bogotanos de Henríquez, Mahecha y Mateus (2016). En cuanto al territorio español, destacamos las investigaciones realizadas en las universidades de Huelva (Prado y Galloso, 2008), Las Palmas de Gran Canaria (Samper, 2009; Samper, Hernández y Samper, 2019, 2020), Navarra (Jiménez, 2015a, 2015b) y Valencia (Gómez-Devís, 2019).

No obstante, aunque se trata de valiosas balizas para el desarrollo más actual, solo comentaremos con detalle aquellos que se centran en el léxico disponible de niños y niñas de 6 años.

En 1993 el investigador mexicano López Chávez publica, junto a un grupo de colaboradores, los repertorios de disponibilidad de los alumnos del nivel primario completo, desde primer grado hasta sexto, a los que se añadió el correspondiente nivel preescolar. Esta contribución abarca los 16 centros de interés tradicionales hasta la fecha y considera un tiempo de tres minutos para cada uno de ellos. En cuanto a los factores sociales atiende al sexo - con el mismo número de niños y niñas- y tipo de centro educativo - escuela oficial/escuela particular-.

Casi al mismo tiempo, en Costa Rica, se desarrolla la investigación de Murillo $(1993,1994)$ con preescolares entre 5 años y medio y 6 años. Esta investigadora consigue 
los datos léxicos a partir de estímulos visuales -láminas motivadoras- y preguntas abiertas como ¿qué es esto? y ¿qué otras (otros) conoces? También limita el tiempo de respuesta en torno a los 3 minutos, pero su estudio solo abarca seis centros de interés y no todos se encuentran entre los dieciséis tradicionales. Hay que esperar hasta 1999 para conocer el inventario de léxico disponible recopilado por Sánchez y Murillo a partir de entrevistas a 108 niños que acuden a centros tanto públicos como privados. En esta ocasión se amplía el número de centros de interés a diez: cuerpo, alimentos, vestido, familia, juegos y juguetes, comunidad, medios de transporte, medios de comunicación, animales, y profesiones y oficios. En 2006 los mismos autores publican los inventarios de léxico disponible definitivos ampliando la muestra del estudio a 216 preescolares.

En los últimos años, Cepeda, Granada y Pomes (2014) analizan los datos de 51 escolares (34 niños y 17 niñas) chilenos de primero de básico de un centro de enseñanza privada de nivel medio de la ciudad de Talca. La investigación, realizada en las instalaciones educativas, abarca 11 centros de interés del proyecto panhispánico (Partes del cuerpo, Ropa y calzado, Alimentos y bebidas, Medios de transporte, Animales, Juguetes, Ciudad, Casa, Escuela, Profesiones y Colores). El tiempo dedicado a cada uno de ellos es de 2 minutos y el entrevistador va anotando las soluciones aportadas durante la encuesta.

En el territorio español solo disponemos de dos investigaciones. La primera, de Samper, Hernández y Samper (2019, 2020), aborda la DL de 40 escolares -mitad chicos y mitad chicas- que cursan primero de primaria en la isla de Las Palmas de Gran Canaria. El lugar de realización de la encuesta de disponibilidad también es el centro educativo y el tiempo se limita a dos minutos por centro de interés. Como en el caso chileno, el entrevistador anota las palabras que el niño va evocando. Asimismo, tal como apuntan los autores, en ocasiones "hubo que favorecer la continuidad de las respuestas con algún estímulo adicional del tipo ¿qué ropa usas cuando hace calor o cuando hace frío? ¿Cuál es tu comida preferida?" (Samper, Hernández y Samper, 2019: 145). Las variables sociales de la muestra grancanaria son sexo, nivel sociocultural de los progenitores y tipo de centro - público o privado/concertado-. En cuanto a los centros de interés investigados, se reducen a cinco: Ropa, Alimentos y bebidas, Escuela, Animales y Colores.

La otra aportación se incardina en la ciudad de Valencia y su área metropolitana. En esta investigación, desarrollada por Gómez-Devís y Llopis, participan 55 escolares 25 niños y 30 niñas- bilingües que cursan primero de Primaria en distintos centros públicos de los que se han seleccionado, como mínimo, cinco alumnos. Las variables manejadas son sexo, nivel sociocultural de los padres, lugar de residencia y lengua habitual (castellano-catalán de Valencia). Los centros de interés observados son 11, los mismos que en la investigación chilena, y al igual que en las dos contribuciones anteriores, el escolar dispone de dos minutos para contestar oralmente. Asimismo, se señala que los entrevistadores son personas conocidas, vinculadas a la comunidad educativa, y que durante la prueba no se anota nada, sino que se esta se graba. Otro aspecto específico de la investigación valenciana es que los encuestados solo pueden recibir algún tipo de ayuda, si es que la necesitan, una vez han superado el primer minuto del tiempo asignado (Gómez-Devís, 2019: 171).

Antes de proseguir con el análisis de los resultados, es necesario motivar la elección de las tres investigaciones ${ }^{6}$ cuyos datos se van a contrastar:

- El tiempo dedicado a registrar las respuestas orales de cada centro de interés es de 2 minutos.

\footnotetext{
${ }^{6}$ Cepeda et al. (2014), Samper et al. (2020) y Gómez-Devís (2019)
} 
- Los criterios de edición de los materiales léxicos recabados han sido los propios del magno Proyecto Panhispánico.

- Al tratarse de encuestas orales es normal que se produzcan repeticiones de algunos términos que, lógicamente se han eliminado.

- La información en torno a las variables sociológicas de los participantes ha sido confirmada posteriormente por los profesores del grupo-clase.

\section{ANÁLISIS Y DISCUSIÓN DE RESULTADOS}

\subsection{DATOS CUANTITATIVOS}

En la Tabla 1 se recogen los resultados relativos al número de palabras aportadas por los escolares de las tres comunidades de habla seleccionadas. En el caso de Gran Canaria y Valencia son relativamente altos los promedios de respuesta de estos niños de solo 6 años, mientras que los chilenos puntúan siempre por debajo. Como era de esperar por los resultados de otros trabajos previos con grupos de estudiantes de mayor edad, es el estímulo "Animales" el que acumula mayor número de respuestas en las tres muestras. No queda muy atrás el centro "Alimentos y bebidas" que obtiene, como es frecuente, promedios más altos que "Ropa" y "Escuela" (Prado y Galloso, 2008; Samper, 2009; Hernández y Samper, 2020).

Tabla 1. Promedio de respuestas por centro de interés en las tres comunidades de habla

\begin{tabular}{lccc}
\hline Centro de & $\begin{array}{c}\text { Chile } \\
\text { (Talca) } \\
\text { Promedio } \\
\text { sujeto }\end{array}$ & $\begin{array}{c}\text { Gran } \\
\text { Canaria } \\
\text { Promedio } \\
\text { sujeto }\end{array}$ & $\begin{array}{c}\text { Valencia } \\
\text { Promedio } \\
\text { sujeto }\end{array}$ \\
\hline Ropa & 9.60 & 16.03 & 13.07 \\
Alimentos & 12.76 & 19.28 & 16.20 \\
Escuela & 11.29 & 17.40 & 15.70 \\
Animales & 12.66 & 20.03 & 19.01 \\
Colores & 12.11 & 17.40 & 14.12 \\
\hline
\end{tabular}

Fuente: Elaboración propia

A continuación, la Tabla 2 presenta los resultados por centro de interés y sexo del escolar en las tres muestras. Solo los niños valencianos aportan mayor número de respuestas que sus compañeras en todos los centros de interés. Las niñas grancanarias, por su parte, superan a sus compañeros en todos los estímulos, salvo en "Animales". En la muestra chilena, las niñas también superan a los niños en cuatro de los cinco estímulos, aunque en esta ocasión es en "Alimentos" donde los varones obtienen mejores resultados.

Tabla 2. Promedio de respuestas por centro de interés y sexo del escolar

\begin{tabular}{lcccccc}
\hline $\begin{array}{l}\text { Centro } \\
\text { de interés }\end{array}$ & $\begin{array}{c}\text { Chile(Talca) } \\
\text { Promedio } \\
\text { niño }\end{array}$ & $\begin{array}{c}\text { Promedio } \\
\text { niña }\end{array}$ & $\begin{array}{c}\text { Gran } \\
\text { Promedio } \\
\text { niño }\end{array}$ & $\begin{array}{c}\text { Canaria } \\
\text { Promedio } \\
\text { niña }\end{array}$ & $\begin{array}{c}\text { Valencia } \\
\text { Promedio } \\
\text { niño }\end{array}$ & $\begin{array}{c}\text { Promedio } \\
\text { niña }\end{array}$ \\
\hline Ropa & 9.18 & 10.29 & 14.40 & 17.65 & 13.15 & 12.76 \\
Alimentos & 13.18 & 11.94 & 18.80 & 19.75 & 17.50 & 15.13 \\
Escuela & 11.15 & 11.53 & 16.95 & 17.85 & 15.93 & 15.63 \\
\hline
\end{tabular}




\begin{tabular}{lllllll}
\hline Animales & 12.47 & 13.06 & 20.50 & 19.55 & 20.93 & 17.60 \\
Colores & 12.03 & 12.29 & 13.80 & 14.75 & 14.40 & 13.90 \\
\hline
\end{tabular}

Fuente: Elaboración propia

El comportamiento de los niños de 6 años se corresponde con el mostrado por la mayoría de los varones en los estudios preuniversitarios al presentar en el estímulo "Animales" el número de respuestas más elevado. En este sentido, Samper y Samper (2006: 30-31) ya constataron unos promedios que oscilan entre las 26.9 y las 27.6 unidades para este estímulo; el más alto de los 16 centros de interés. Asimismo, "Alimentos y bebidas" suele ocupar el segundo lugar con unas medias generales que fluctúan entre las 25,5 y las 26,2 unidades. Por último, en cuanto a "Ropa", que siempre es uno de los centros que visibiliza la distinción entre mujeres y hombres, siendo las primeras las que muestran mejores resultados, en esta ocasión no es tan evidente.

\section{2. ORGANIZACIÓN DEL LÉXICO EN LA MENTE: REDES}

Desde una vertiente cognitiva esta prueba asociativa de carácter artificial ofrece datos léxicos fruto de un proceso de activación regulado por una red $^{7}$ ilimitada de asociaciones semánticas, fonéticas, personales, culturales, etc. En este sentido, resulta evidente que proporcionar la lista de palabras más disponibles ${ }^{8}$ de un determinado estímulo no es una opción. Se requiere una representación de las unidades léxicas disponibles y las múltiples relaciones que han establecido entre sí. Para ello disponemos de un programa computacional denominado DispoGrafo, diseñado por investigadores de la Universidad de Concepción (Chile), que proporciona valiosos datos.

Utilizando un algoritmo basado fundamentalmente en las relaciones de secuencia de las palabras disponibles, nuestro programa, DispoGrafo, ingresa los términos y genera luego automáticamente grafos cuyos nodos representan palabras y cuyas aristas simbolizan las relaciones entre ellas. [...] Los grafos se interpretan como redes semánticas cuya configuración expresa las relaciones semánticas subyacentes en el corpus. El software permite, además, eliminar las conexiones débiles (de menor peso) y visualizar de este modo las relaciones más relevantes.

(Echeverría et al., 2008: 81)

En otras palabras, este programa permite observar las relaciones asociativas unidad léxicas encadenadas en las listas producidas por los informantes- de todas las palabras que conforman el inventario disponible de un centro de interés. Es capaz de mostrar con detalle cuáles han sido las relaciones que se han presentado con mayor o menor frecuencia e incluso los vínculos indirectos y pequeñas organizaciones categoriales. Ello nos facilita enormemente la tarea de establecer el tipo de conexiones significativas y léxicas que sostienen dichas configuraciones -redes- en cualquier estímulo observado.

Así pues, asumimos la necesidad de indagar las categorías o subcategorías semánticas representadas en un centro de interés, qué unidades léxicas las integran o cuál es el volumen de relaciones establecidas entre aquellas que muestran mayor conectividad. A modo de ejemplo, la imagen que aparece a continuación muestra con excelente detalle la organización del léxico disponible de los valencianos de 6 años en el centro de interés

\footnotetext{
${ }^{7}$ El término "red" no se emplea en sentido metafórico, ya que neuronalmente esa es su estructura. La neurología está confirmando que los hechos del lenguaje se construyen mediante redes neuronales sinápticas.

${ }^{8}$ Se recuerda que el cálculo del índice de disponibilidad (ID) se basa en la frecuencia de aparición en los listados y la posición que ocupa.
} 
“Animales”. Para una interpretación más ajustada se recuerda al lector que los nodos representan las unidades léxicas disponibles y las aristas simbolizan las relaciones que se han establecido entre ellas -la flecha marca el sentido mayoritario de las conexiones y el número que aparece sobre ella indica el total de conexiones establecidas-. Para esta ocasión se han eliminado las unidades que no se han relacionado con otras al menos en dos ocasiones ${ }^{9}$.

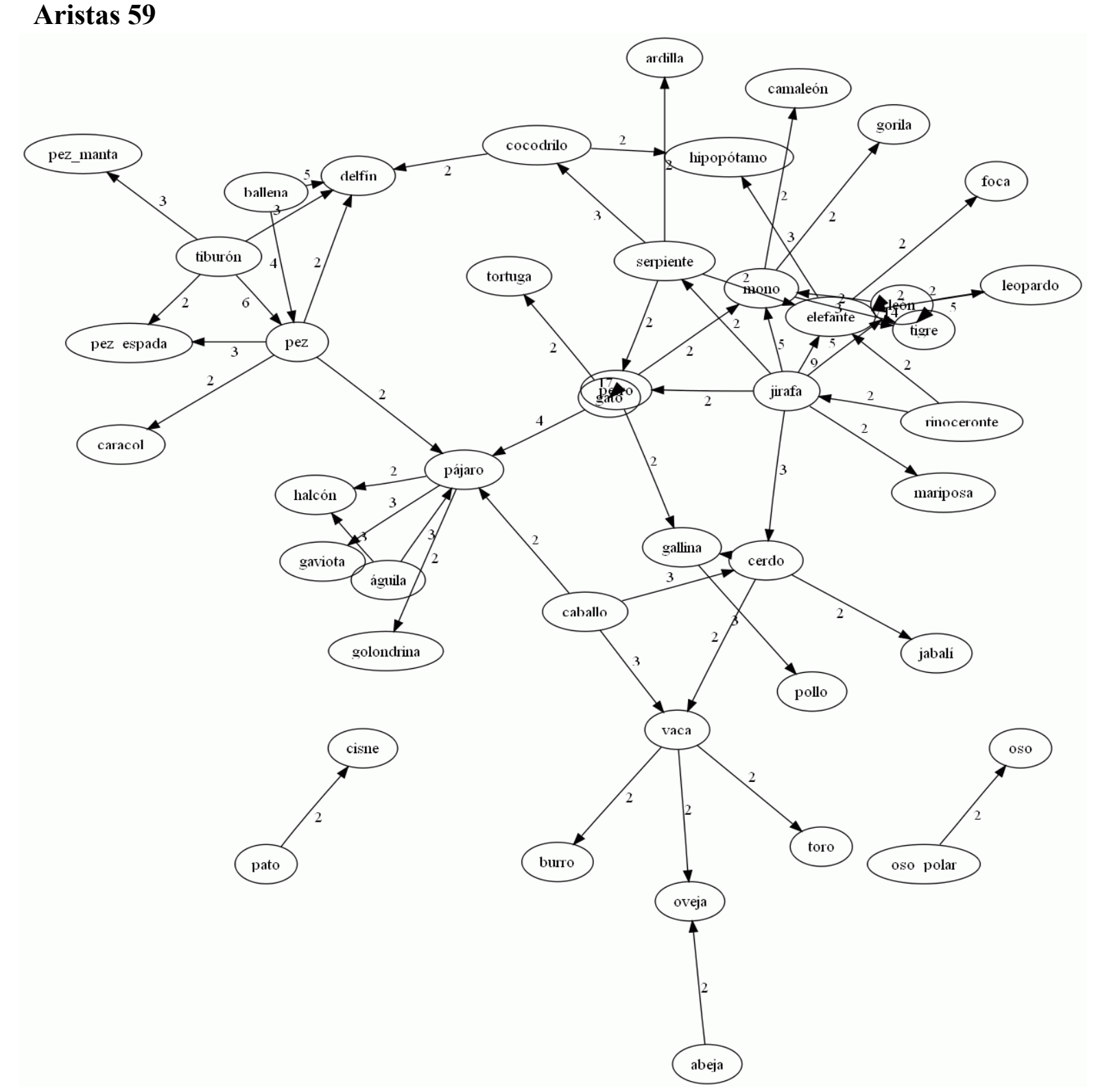

Imagen 1. Grafo general de los niños valencianos de 6 años. Nodos 44 y Aristas 59

El grafo de la Imagen 1 permite observar con claridad la distribución jerárquica y categorial de las palabras evocadas e interconectadas, al menos dos ocasiones, por los 55 escolares valencianos. Se aprecia que las unidades prototípicas -zona central de la

${ }^{9}$ Hay once unidades léxicas procedentes del catalán -variedad valenciano- registradas en el inventario disponible pero no aparecen en el grafo porque que el número de conexiones entre dichos términos y el resto de unidades disponibles no ha superado la cifra de al menos dos ocasiones. No obstante, los participantes que las han verbalizado sí superan esta cifra. 
imagen- son perro $\rightarrow$ gato con 17 conexiones; seguidas de león $\rightarrow$ tigre (14); tigre $\rightarrow$ elefante (7); y león $\rightarrow$ leopardo (5).

Asimismo, la distribución de los nodos - palabras- puede relacionarse con distintos subconjuntos:

- animales de la selva: león, tigre, leopardo, elefante, jirafa, mono, gorila, camaleón, hipopótamo, cocodrilo, rinoceronte y serpiente;

- animales de granja: cerdo, caballo, vaca, gallina, pollo, burro, toro, jabalí;

- animales marinos: pez, tiburón, ballena, delfín, pez manta, pez espada, caracol; y por último,

- animales que vuelan: pájaro, gaviota, halcón, águila y golondrina.

También es posible apreciar el valor facilitador de un nodo sobre otro por distintas razones: similitud de significantes, abeja $\rightarrow$ oveja (2); mecanismo de hipónimo e hiperónimo, oso polar $\rightarrow$ oso (2); y semejanza de significado, pato $\rightarrow$ cisne (2) o cerdo $\rightarrow$ jabalí (2). Sin olvidar, además, la precisión que nos proporciona sobre el término caracol -marino-.

La utilización de los grafos para analizar la activación del léxico disponible y los mecanismos de asociación ya es un hecho contrastado (Henríquez, Mahecha y Mateus, 2016; Mahecha y Mateus, 2017). En este sentido, se ha comentado que el programa DispoGrafo también puede mostrar las relaciones que se han establecido entre las palabras actualizadas según las distintas variables sociológicas. A modo de ejemplo, la Imagen 2 muestra los grafos de acuerdo con una distribución sexo/género. Dicha imagen nos permite apreciar que, si bien los niños han aportado mayor número de unidades léxicas que sus compañeras, estas no solo han logrado manifestar un sistema de redes más amplio, sino que, además, han sido capaces de establecer mayor número de conexiones entre las unidades léxicas -redes más fuertes-. Los datos recabados así lo confirman: los niños cuentan con 26 unidades léxicas conectadas en dos ocasiones o más y 23 aristas, y las niñas han logrado 28 nodos y 30 aristas. También es interesante señalar que al observar las unidades que mayor conectividad han mantenido entre sí no hay coincidencia en los subgrupos. Para las chicas sigue siendo perro-gato (12 conexiones), como en los datos de la muestra general, mientras que para los chicos es león-tigre (11 conexiones).

\section{sexo/género}

Imagen 2. Grafo de los niños valencianos de 6 años según la variable

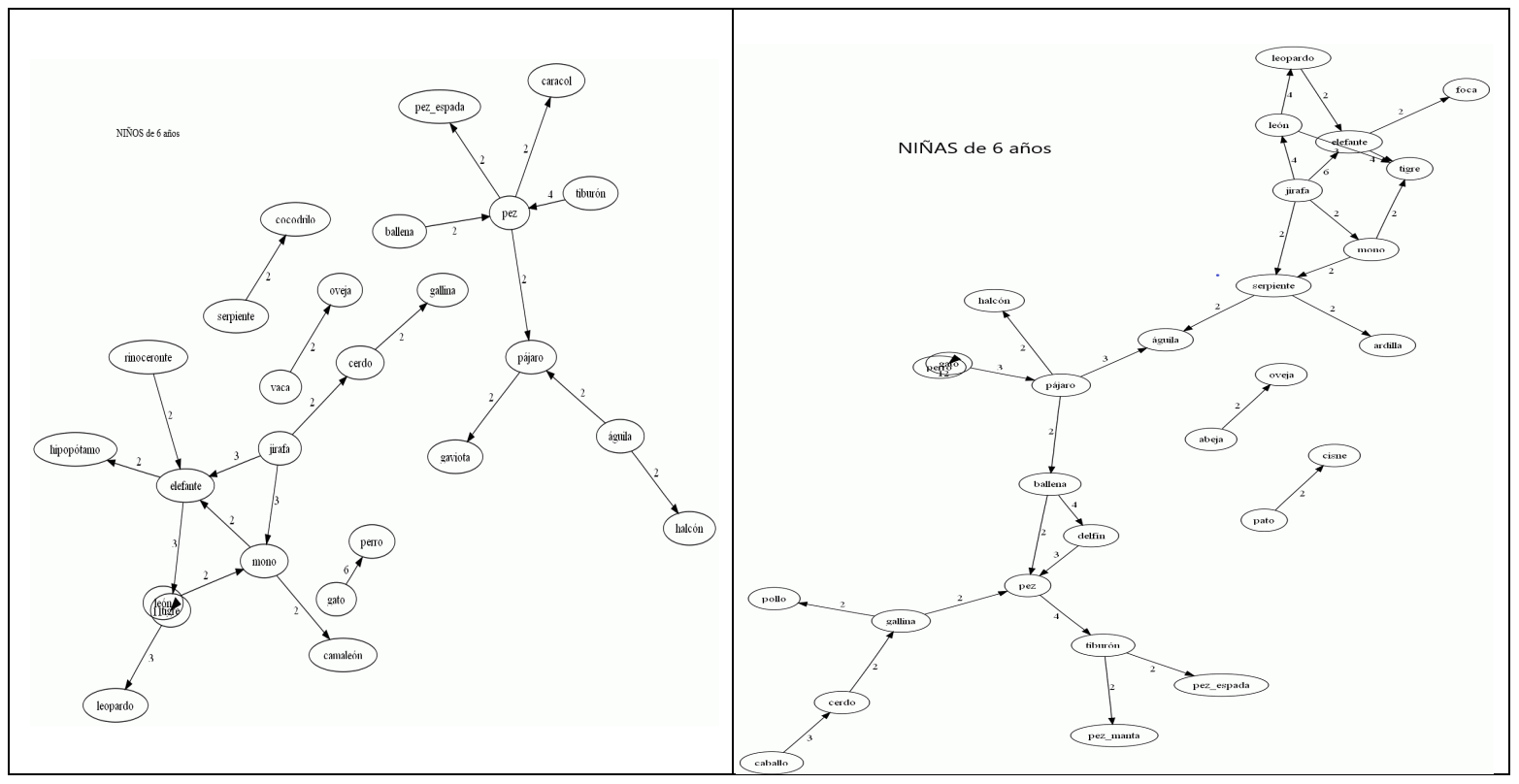




\section{CONCLUSIONES}

A lo largo de esta contribución se ha puesto de manifiesto que el abordaje de la DL en edades tempranas (6 años) abre nuevas perspectivas y ricas posibilidades de aplicación al terreno de la enseñanza. En cuanto a la proyección de sus resultados, no solo resulta muy valioso el análisis de los datos cuantitativos y su comparación con otras investigaciones -progresión cuantitativa en distintos niveles educativos, promedios por informante, total de vocablos por centro de interés, etc.-, también es muy útil determinar el carácter de las relaciones asociativas que han manifestado los niños y niñas, así como distinguir entre las que son de naturaleza lingüística y las de naturaleza extralingüística. De las primeras interesan especialmente las relaciones léxicas, ya sean auténticas relaciones semánticas desde el significado (sinonimia, antonimia, hiperonimia, hiponimia), ya sean pseudorrelaciones semánticas a partir del significante (polisemia y homonimia). Las extralingüísticas, también denominadas "significativas", se establecen mediante cualquier tipo de vínculo basado en el contenido (metáfora, metonimia, etimología popular, relaciones de contigüidad o meronimia, terminologías, asociaciones de carácter cultural o experiencial, etc.)

Por otro lado, es evidente que la trascendencia de los resultados aquí manejados todavía se encuentra en fase incipiente, especialmente si los comparamos con los obtenidos en estudiantes preuniversitarios, pero el objetivo a corto plazo es ahondar, ahora desde el ámbito infantil, en el marco de las lenguas primeras y segundas (Hernández y Tomé, 2017; Gómez-Devís y Llopis, 2016; Serrano, 2020) o del futuro profesorado (Santos, 2017, 2020; Santos, Trigo y Romero, 2020).

En definitiva, descubrir el bagaje léxico de los escolares de cualquier edad es el primer paso para conseguir ampliarlo; esto es, comunicarse con mayor precisión, propiedad y claridad, así como propiciar procesos mentales tan importantes como ordenar, relacionar, matizar o clasificar la realidad individual y extrapersonal. Abordar su enseñanza a partir de las relaciones que los hablantes establecen entre las palabras de cada centro de interés es un asunto palmario.

\section{REFERENCIAS BIBLIOGRÁFICAS}

Aichitson, I. 1987. Words in the mind: an introduction to the mental lexicon, Oxford: Basil Blackwell, 1995.

Cepeda, M., Granada, M., y Pomes, M. 2014. «Disponibilidad léxica en estudiantes de primero básico», Literatura y Lingüística, 30: 181-205. http://dx.doi.org/10.4067/S0716-58112014000200010

Dimitrijević, N. (1969). Lexical Availability. A new aspect of the lexical availability of secondary school children. Heidelberg: Julius Gross Verlag.

Echeverría, M. 1991. "Crecimiento de la disponibilidad léxica en estudiantes chilenos de nivel básico y medio». En La enseñanza del español como lengua materna, ed. López Morales. Río Piedras: Universidad de Puerto Rico, 61-78.

Echeverría, M., Vargas, R., Urzúa, P y Ferreira, R. 2008. «Dispografo: una nueva herramienta computacional para el análisis de relaciones semánticas en el léxico disponible». Revista de Lingüística teórica y aplicada, 46 (1): 81-91.

Ellis, N. 2011. «La memoria para la lengua». En La dimensión cognitiva en la enseñanza de lenguas, ed. Robinson. Madrid: Edinumen, 41-74. 
Gómez-Devís, M-B. 2019. «A propósito de las redes semánticas en el léxico disponible de escolares de primero de Educación Primaria», Ogigia, 25: 165-183. https://doi.org/10.24197/ogigia.25.2019.165-183

Gómez-Devís, M-B. y Llopis, F. 2016. «Xarxes semàntiques en el lèxic disponible de València: una aproximació al lexicó dels bilingües». Studia Románica Posnaniensia, 43 (2): 65-83. https://doi.org/10.14746/strop.2016.432.004

Gougenheim, G., Michéa, R., Rivenc, P., y Sauvageot, A. 1956. L'élaboration du francais élémentaire. Paris: Didier.

Gougenheim, G., Michéa, R., Rivenc, P., y Sauvageot, A. 1964. L'élaboration du francais fondamental (I degré). Étude sur l'élaboration d'un vocabulaire et d'une grammaire de base. Paris: Didier.

Henríquez, Ma C., Mahecha, V., y Mateus, G. 2016. «Análisis de los mecanismos cognitivos del léxico disponible del cuerpo humano a través de grafos». Lingüistica y Literatura, 69: 229-251. https://doi.org/10.17533/udea.lyl.n69a10

Hernández Solís, M ${ }^{a}$ M. 2014. De la palabra al vocablo hay un buen trecho. Disponibilidad léxica de estudiantes de primaria de la ciudad de Zacatecas. Universidad Autónoma de Zacatecas.

Hernández, N., y Tomé, C. 2017. «Léxico disponible en primera y segunda lengua: bases cognitivas». En Palabra, Vocabulario, Léxico. La lexicología aplicada a la didáctica y a la diacronía, ed. Del Barrio De la Rosa. Venezia: Università Ca' Foscari, 99-122.

Herranz Llácer, C. 2019. «Estado actual de los estudios de disponibilidad léxica en España». LEA: Lingüística Española Actual, 41 (I): 93-130.

Hunt, R., y Ellis, H. 2004. Fundamentals of cognitive psycology. Boston: McGraw Hill, ( 7 th edition).

Jiménez, F. 2015a. El léxico disponible de escolares navarros de primaria y secundaria obligatoria. Tesis doctoral. Universidad de Navarra.

Jiménez, F. 2015b. «El léxico disponible de estudiantes navarros de Educación Primaria y ESO. Implicaciones para la enseñanza de vocabulario». En Tendencias en educación lingüística, ed. Cancelas Ouviña. Granada: GEU Editorial, 85-102.

Llopis, F., y Gómez-Devís, M-B. 2010. El lèxic disponible de València. Valencia: Denes.

López Chávez, J., y Strassburger. C. 1991. «Un modelo para el cálculo de disponibilidad léxica individual». En La enseñanza del español como lengua maternal, ed. López Morales. Río Piedras: Universidad de Puerto Rico, pp. 91-112.

López Chávez, J. 1993. El léxico disponible de escolares mexicanos. colección de siete cuadernos (preescolar y primaria). México: Alhambra mexicana.

Lopez, B. y Álvarez de la Granja, M. 2014. Léxico dispoñible do galego, Verba, Anexo 71.

López Morales, H. (1973). Disponibilidad léxica en escolares de San Juan. San Juan de Puerto Rico.

López Morales, H. 1986. La enseñanza de la lengua materna. Lingüistica para maestros de español. (2. ${ }^{\mathrm{a}}$ ed). Madrid: Playor.

Mahecha, V., y Mateus, G. 2017. «El léxico disponible y sus mecanismos de asociación: un análisis con grafos». En Palabra, Vocabulario, Léxico. La lexicología aplicada a la didáctica y a la diacronía, ed. Barrio de la Rosa. Venezia: Università Ca' Foscari, 123-142.

Murillo, M. 1993. «Disponibilidad léxica en los preescolares: estudio de cinco campos semánticos». Káñima, Revista Artes y Letras, XVII (2): 117-127.

Murillo, M. 1994. «Comidas y bebidas: estudio de la disponibilidad léxica en preescolares». Káñima, Revista. Artes y Letras, XVIII (2): 117-133. 
Paredes, F. 2012. «Desarrollos teóricos y metodológicos recientes de los estudios de disponibilidad léxica». Revista Nebrija de Lingüística Aplicada, 11(6): 78-100.

Prado, J., y Galloso, M ${ }^{\mathrm{a}}$ V. 2008. Léxico disponible de Huelva. Nivel $6^{\circ}$ de Educación Primaria. Huelva: Universidad de Huelva.

Samper, M. 2009. Evolución de la disponibilidad léxica en estudiantes grancanarios de enseñanza primaria y secundaria. Las Palmas de Gran Canaria: Cabildo de Gran Canaria.

Samper, M., Hernández, C., y Samper, J. A. 2020. «Incidencia del factor sexo/género en la disponibilidad léxica de niños grancanarios de 6 años». En Lengua, sociedad y cultura. Estudios dedicados a Alberto Carcedo, ed. Gimeno Menéndez. Alicante: Universitat d'Alacant, pp. 287-312.

Samper, J. A.; Hernández, C. y Samper, M. 2019. «Disponibilidad léxica en niños grancanarios de 6 años. Comparación con México y Costa Rica». Ogigia, 25: 139163. https://doi.org/10.24197/ogigia.25.2019.139-163

Samper, J. A. y Samper, M. (2006). «Aportaciones recientes de los estudios de disponibilidad léxica». LynX: Panorámica de estudios Lingüísticos, 5: 5-95.

Sánchez, V. y Murillo, M. 1999. "Inventario del léxico disponible de los preescolares costarricenses». Káñima, Rev. Artes y Letras, XXIV (I): 103-136.

Sánchez, V. y Murillo, M. 2006. Disponibilidad léxica de los niños preescolares costarricenses. San José: Universidad de Costa Rica.

Santos, I. C. 2020. El léxico bilingüe del futuro profesorado. Análisis y pautas para estudios de disponibilidad léxica. Berlin: Peter Lang.

Santos, I. C. 2017. «Organización de las palabras en la mente en lengua materna y lengua extranjera (inglés y francés)». Pragmalingüistica, 25: 603-617.

Santos, I. C., Trigo, E. y Romero, M. F. 2020. «La activación del léxico disponible y su aplicación a la enseñanza de lenguas». Porta Linguarum, 33: 75-93.

Serrano, M. 2014. Disponibilidad léxica en la provincia de Lleida: estudio comparado de dos lenguas en contacto. Tesis doctoral. Universitat de Lleida.

Serrano, M. 2020. «Disponibilidad léxica en las comunidades bilingües de España». En Lengua, sociedad y cultura. Estudios dedicados a Alberto Carcedo, ed. Gimeno Menéndez. Alicante: Universitat d'Alacant, pp. 313-334. 